Research Article

\title{
Load Frequency Control for Power Systems with Actuator Faults within a Finite-Time Interval
}

\author{
Haifeng Qiu (D), Liguo Weng, Bin Yu, and Yanghui Zhang \\ State Grid Hangzhou Xiaoshan Power Supply Company, Hangzhou 311200, China \\ Correspondence should be addressed to Haifeng Qiu; 13857177475@sina.cn
}

Received 14 August 2021; Accepted 21 October 2021; Published 8 November 2021

Academic Editor: Meng Li

Copyright ( $\odot 2021$ Haifeng Qiu et al. This is an open access article distributed under the Creative Commons Attribution License, which permits unrestricted use, distribution, and reproduction in any medium, provided the original work is properly cited.

\begin{abstract}
This paper is concerned with the issue of finite-time $H_{\infty}$ load frequency control for power systems with actuator faults. Concerning various disturbances, the actuator fault is modeled by a homogeneous Markov chain. The aperiodic sampling data controller is designed to alleviate the conservatism of attained results. Based on a new piecewise Lyapunov functional, some novel sufficient criteria are established, and the resulting power system is stochastic finite-time bounded. Finally, a single-area power system is adjusted to verify the effectiveness of the attained results.
\end{abstract}

\section{Introduction}

Load frequency control (LFC), as an integral part of automatic generation control in power systems, has been adopted to regulate the frequency deviation and tie-line power exchanges [1-3]. Added by the LFC strategy, the high-quality electric energy can be maintained over a certain range [4]. In general, constant frequency deviation may lead to unreliable frequency devices, transmission lines overload, etc. Meanwhile, owing to the large size of the power grid, it raises the difficulty in frequency control. Therefore, it is a tough task to design suitable frequency control law. In practical applications, the loads are unexpected and unmeasurable, which indirectly regulate the system frequency. Accordingly, through the LFC strategy, the system performance can be guaranteed without affecting the generation capacity or frequency deviation. Up to now, the research on the LFC for power system gradually becomes a hot topic [5-7].

In networked control systems, various faults can be encountered due to the long-term utilization of components [8-10]. Note that the actuator faults are the source of instability and performance deterioration. To overcome the above shortage and improve the dependability, a great deal of attention has been shifted to actuator faults, and plenty of results have emerged $[11,12]$. However, the actuator faults are assumed to be time-unchanged, which limits the potential applications. As stated in [13], the so-called failure probability is common in the reliability industry, where failure rates can be governed by the Markov switching chain [14-16]. Despite the significant achievement has attained, no suitable attention has been devoted to the power systems.

On the other hand, Lyapunov asymptotic stability is most common in the literature, where asymptotic behavior can be expected over the infinite-time domain. Nevertheless, in reality, the desirable transient performance is very important in many physical systems, which causes the inapplicability of the Lyapunov stability. Following this trend, finite-time stability (FTS) has been studied [17-19], which concerns the dynamic behavior within bound over a fixed time interval instead of an asymptotical case. As is well known that FTS is different from the Lyapunov case, it gives more solutions of transient performance control. Owing to the merits of the FTS, many valuable achievements have been made over the past years [20]. However, to our knowledge, most of the previous results are assumed that the data communication keeps continuous between sensors and controllers. In the fields of sampled-data control law, this assumption is not accurate. In general, with respect to the demand of actual systems, the sampler may encounter component aging, data losses, etc. [21-23]. These shortages may lead to unreliable periodic sampling. Fortunately, the aperiodic sampled-data control strategy is presented 
$[24,25]$, which can efficiently deal with the aforementioned issues. However, the finite-time aperiodic sampled-data control for power systems remains unsettled, not mentioned to the LFC, which motivates us for this study.

Inspired by the above observations, we focus on the finite-time $H_{\infty}$ load frequency control for power systems with actuator faults over the finite-time interval in this study. The main contributions can be summarized as follows: (1) different from the previous studies, to fully describe the randomly occurring actuator fault, the actuator fault is characterized by a homogeneous Markov chain. (2) To better characterize the actual demands of practical dynamics, a generalized framework of the actuator constraint is considered. (3) Apart from the traditional Lyapunov asymptotic stability, this study exploits the FTS for power systems and focuses on the finite-time control issue. By resorting to the piecewise Lyapunov theory, some novel results over the finite-time interval are reached. Finally, a numerical example is manifested to reveal the validity of the gained results.

The remainder of this study is listed as follows. Section 2 provides a description of the problem. Section 3 presents the main results, and the simulation validation is exhibited in Section 4 . Section 5 concludes the study.

1.1. Notations. The notations of this paper are standard. $\|\cdot\|$ means the Euclidean norm. $\mathbb{R}$ indicates a set of $n$-dimensional matrix. $E$ refers to the mathematical expectation. $\left(\lambda_{\max }(\mathrm{A}) / \lambda_{\min }(\mathrm{A})\right)$ means the largest/smallest eigenvalue of matrix A. $\operatorname{Pr}\{\cdot\}$ means the occurrence probability. diag $\{\cdot\}$ represents a block-diagonal matrix.

\section{Problem Formulations}

Block diagram of single-area LFC power model is exhibited in Figure 1 [6]. Accordingly, the dynamic equation of power model can be listed as follows:

$$
\left\{\begin{array}{l}
\dot{\bar{\delta}}(t)=\bar{A} \bar{\delta}(t)+\bar{B} u^{F}(t)+\bar{F} \omega(t), \\
\bar{y}(t)=\bar{C} \bar{\delta}(t),
\end{array}\right.
$$

where

$$
\begin{aligned}
\bar{\delta}(t) & =\left[\begin{array}{lll}
\Delta f & \Delta P_{m} & \Delta P_{v}
\end{array}\right]^{T}, \\
\omega(t) & =\Delta P_{d}, \\
\bar{C} & =\left[\begin{array}{lll}
\beta & 0 & 0
\end{array}\right], \\
\bar{A} & =\left[\begin{array}{ccc}
-\frac{D}{M} & \frac{1}{M} & 0 \\
0 & -\frac{1}{T_{c h}} & \frac{1}{T_{c h}} \\
-\frac{1}{R T_{g}} & 0 & -\frac{1}{T_{g}}
\end{array}\right], \\
\bar{B} & =\left[\begin{array}{lll}
0 & 0 & \frac{1}{T_{g}}
\end{array}\right]^{T}, \\
\bar{F} & =\left[\begin{array}{lll}
-\frac{1}{M} & 0 & 0
\end{array}\right]^{T},
\end{aligned}
$$

and the system parameters are expressed in Table 1.

In single-area, the area control error (ACE) is interpreted as $y(t)=\beta \Delta f$ due to the unaccessiblity of the tie-line power exchange. In reality, the actuator faults cannot be neglected for long-term utilization of components, which can be expressed as

$$
u^{F}(t)=\alpha\left(r_{t}\right) u(t)
$$

where $\alpha\left(r_{t}\right)=\operatorname{diag}\left\{\alpha_{1}\left(r_{t}\right), \alpha_{2}\left(r_{t}\right), \ldots, \alpha_{f}\left(r_{t}\right)\right\}$, and each element $\alpha_{m}\left(r_{t}\right) \in[0,1](m=1,2, \ldots, f)$. More specifically, $\left\{r_{t}, t \geq 0\right\}$ is identified as a right-continuous Markov chain taking values over a set $\mathcal{S}=\{1,2, \ldots, S\}$ with generator $\Pi=\left[\pi_{p q}\right]_{S \times S}$, and its transition probabilities are inferred as

$$
\operatorname{Pr}\left\{r_{t+\Delta t}=q \mid r_{t}=p\right\}= \begin{cases}\pi_{p q} \Delta t+o(\Delta t), & \text { if } q \neq p, \\ 1+\pi_{p q} \Delta t+o(\Delta t), & \text { if } q=p,\end{cases}
$$

where $\Delta t>0$ and $\left(\lim _{\Delta t \rightarrow 0} o(\Delta t) / \Delta t\right)=0$, for $q \neq p$ and $\pi_{p p}=-\sum_{q \neq p} \pi_{p q}$ for each $p \in \mathcal{S}$.

Taking the ACE as the desired controller input of LFC, the output of the proportional-integral (PI) controller is asserted as

$$
u(t)=-K_{P} \bar{y}(t)-K_{I} \int \bar{y}(t),
$$

where $K_{P}$ and $K_{I}$ signify the proportional and integral gains of the area, respectively.

Let $\delta(t)=\left[\bar{\delta}^{T}(t) \int_{0}^{t} \bar{y}^{T}(s) \mathrm{d} s\right]^{T}$, $y(t)=\left[\bar{y}^{T}(t) \int_{0}^{t} \bar{y}^{T}(s) \mathrm{d} s\right]^{T}$, the power model (1) is reformulated as

$$
\left\{\begin{array}{l}
\dot{\delta}(t)=A \delta(t)+B \alpha\left(r_{t}\right) u(t)+F \omega(t), \\
y(t)=C \delta(t)
\end{array}\right.
$$

where

$$
A=\left[\begin{array}{cccc}
-\frac{D}{M} & \frac{1}{M} & 0 & 0 \\
0 & -\frac{1}{T_{c h}} & \frac{1}{T_{c h}} & 0 \\
-\frac{1}{R T_{g}} & 0 & -\frac{1}{T_{g}} & 0 \\
\beta & 0 & 0 & 0
\end{array}\right],
$$

$$
B=\left[\begin{array}{c}
0 \\
0 \\
\frac{1}{T_{g}} \\
0
\end{array}\right],
$$




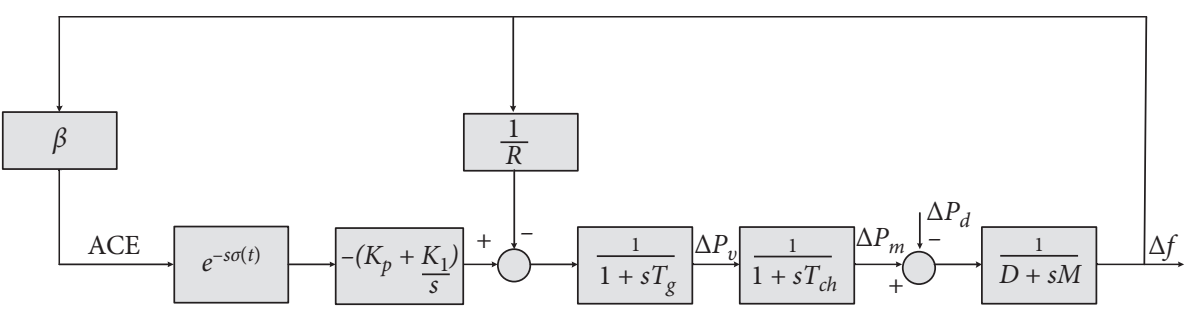

Figure 1: The structure of single-area power system.

$$
\begin{aligned}
& F=\left[\begin{array}{c}
-\frac{1}{M} \\
0 \\
0 \\
0
\end{array}\right], \\
& C=\left[\begin{array}{llll}
\beta & 0 & 0 & 0 \\
0 & 0 & 0 & 1
\end{array}\right] .
\end{aligned}
$$

The purpose of this study is to solve the output feedback control for power system (6) with data sampling. Therefore, the sampling sequence attained at a set of time instants. Added by the data sampling technique, only the measured signal $y\left(t_{k}\right)$ can be released to the controller. Specifically, the sampling instants are represented as

$$
0=t_{0}<t_{1}<\cdots<t_{k}<t_{k+1}<\cdots<\lim _{k \longrightarrow \infty} t_{k}=\infty .
$$

In light of periodic sampling instants, in this study, we consider the aperiodic sampling case. Following this trend, the sampling interval $\left[t_{k}, t_{k+1}\right)$ is time-varying with the upper sampling period. Thus, one defines $0<t_{k+1}-t_{k} \triangleq \tau_{k} \leq \tau$. Based on the input delay technique, we have that $t_{k}=t-\left(t-t_{k}\right) \triangleq t-\tau(t)$ with $\dot{\tau}(t)=1$ for $t \neq t_{k}$. Summarizing the above discussion, we have $0 \leq \tau(t)<\tau_{k} \leq \tau$.

Letting $K=\left[\begin{array}{ll}K_{p} & K_{I}\end{array}\right]$, the PI-based sampled data LFC can be designed as

$$
u(t)=K y\left(t_{k}\right), \quad t \in\left[t_{k}, t_{k+1}\right) .
$$

Substituting (3) and (9) into (6), the closed loop power system can be governed by

$$
\dot{\delta}(t)=A \delta(t)+B \alpha\left(r_{t}\right) K C \delta\left(t_{k}\right)+F \omega(t), \quad t \in\left[t_{k}, t_{k+1}\right) .
$$

Before further derivation, some important contents are stated as follows.

Assumption 1 (see [18]). The external disturbance $\omega(t)$ belongs to $L_{2}\left[t_{0}, T\right)$, and it is assumed that there exists a parameter $\bar{\omega}>0$ such that $\int_{t_{0}}^{T} \omega^{T}(t) \omega(t) \mathrm{d} t \leq \bar{\omega}$.
Table 1: Physical meaning.

\begin{tabular}{lc}
\hline Parameters & Physical meaning \\
\hline$\Delta P_{v}$ & Governer valve position \\
$\Delta f$ & Deviations of frequency \\
$\Delta P_{m}$ & Mechanical output of generator \\
$\beta$ & Frequency bias factor \\
$\Delta P_{d}$ & Load disturbance \\
$M$ & Moments of inertia of the generator \\
$R$ & Speed drop \\
$T_{d}$ & Time constant of the governor \\
$D$ & Damping coefficient of generator \\
$T_{c h}$ & Time constant of turbine \\
\hline
\end{tabular}

Assumption 2. From the viewpoint of the physical limitation of actuators in power systems, the control torque is assumed to meet

$$
\left|u_{s}(t)\right| \leq u_{\max }, \quad s=1,2, \ldots, n_{u} .
$$

Definition 1 (see [26]). Given parameters $c_{1}>0, c_{2}>0$, time interval $T>0$, and matrix $R>0$, the closed loop power system (10) is called stochastic finite-time stability (SFTS) with respect to $\left(c_{1}, c_{2}, T, R\right)$ and $\omega(t)=0$, if inequality $\mathrm{E}\left\{\delta^{T}\left(t_{0}\right) R \delta\left(t_{0}\right)\right\} \leq c_{1} \Rightarrow \mathrm{E}\left\{\delta^{T}(t) R \delta(t)\right\} \leq c_{2}$ holds for $\forall t \in\left[t_{0}, T\right]$.

Definition 2 (see [26]). Given parameters $c_{1}>0, c_{2}>0, \bar{\omega}>0$, time interval $T>0$, and matrix $R>0$, the closed loop power system (10) is called stochastic finite-time boundedness (SFTB) with respect to $\left(c_{1}, c_{2}, T, R, \bar{\omega}\right)$, if inequality $\mathrm{E}\left\{\delta^{T}\left(t_{0}\right) R \delta\left(t_{0}\right)\right\} \leq c_{1} \Rightarrow \mathrm{E}\left\{\delta^{T}(t) R \delta(t)\right\} \leq c_{2}$ holds for $\forall t \in\left[t_{0}, T\right]$.

The object of this study is to design sampled-data-based controller (9) such that

(1) The closed-loop power system (10) is SFTS with $\omega(t)=0$.

(2) When disturbances $\omega(t) \neq 0$, the power system (10) is called SFTB with $H_{\infty}$ performance index $\gamma$ such that

$$
\mathrm{E}\left\{\|y(t)\|_{2}^{2}\right\}<\gamma^{2}\|\omega(t)\|_{2}^{2}
$$

Lemma 1 (see [27]). For any vectors $\hbar_{1}(t)$ and $\hbar_{2}(t)$, scalar $\iota \in(0,1)$, a matrix $\mathrm{A}$, and symmetric matrices $\mathrm{B}$ and $\mathrm{C}$, the following inequality holds: 


$$
-\frac{1}{l} \hbar_{1}^{T}(t) \mathrm{B} \hbar_{1}(t)-\frac{1}{1-l} \hbar_{2}^{T}(t) \mathrm{C} \hbar_{2}(t) \leq-\left[\begin{array}{c}
\hbar_{1}(t) \\
\hbar_{2}(t)
\end{array}\right]^{T}\left[\begin{array}{ll}
\mathrm{B} & \mathrm{A} \\
* & \mathrm{C}
\end{array}\right]\left[\begin{array}{l}
\hbar_{1}(t) \\
\hbar_{2}(t)
\end{array}\right] .
$$

\section{Main Results}

Theorem 1. For given parameters $\rho>0, c_{1}>0, c_{2}>0, T>0$, $\bar{\omega}>0, u_{\max }$, and matrix $R$, the closed-loop power system (10) is called SFTB with respect to $\left(c_{1}, c_{2}, \bar{\omega}, T, R\right)$, if there exists matrix $P_{p}>0, N_{1}>0, N_{3}>0, Q_{1}>0$, and $Q_{2}>0$, such that $\forall p \in \mathcal{S}$

$$
\begin{gathered}
Q_{f} \geq 0, \quad f=1,2, \\
\Theta^{1}<0,
\end{gathered}
$$

$$
\begin{gathered}
{\left[\begin{array}{cc}
\Theta^{2} & \tau_{p}^{T} \mathscr{H}_{p}^{T} \\
* & -\tau Q_{1}
\end{array}\right]<0,} \\
\psi c_{1}+\gamma^{2} \bar{\omega}<e^{-\rho T} \min _{p \in \mathcal{S}}\left\{\lambda_{\min }\left(\widetilde{P}_{p}\right)\right\} c_{2}, \\
{\left[\begin{array}{cc}
-u_{s \max }^{2} & C^{T} K_{s}^{T} \\
* & -\frac{R}{c_{2}}
\end{array}\right]<0, \quad s=1,2, \ldots, n_{u} .}
\end{gathered}
$$

where

$$
\begin{aligned}
& \Theta^{i}=\left[\begin{array}{ccccc}
\Theta_{11}^{i} & \Theta_{12}^{i} & \Theta_{13} & \Theta_{14}^{i} & \Theta_{15} \\
* & \Theta_{22}^{i} & H_{8}^{\top} & \Theta_{24}^{i} & H_{10} \\
* & * & \Theta_{33} & 0 & 0 \\
* & * & * & \Theta_{44}^{i} & T_{2}^{\top} F \\
* & * & * & * & -\gamma^{2} I
\end{array}\right], \\
& Q_{1}=\left[\begin{array}{cc}
N_{1} & U \\
* & N_{1}
\end{array}\right] \\
& Q_{2}=\left[\begin{array}{cc}
Q_{2} & V \\
* & Q_{2}
\end{array}\right] \\
& \Theta_{11}^{1}=\sum_{q=1}^{S} \pi_{p q} P_{q}-M_{1}+Q_{1}-Q_{2}+T_{1}^{T} A+A^{T} T_{1}-\rho P_{p}-\tau \rho M_{1}-H_{1}-H_{1}^{T} \text {, } \\
& \Theta_{11}^{2}=\sum_{q=1}^{S} \pi_{p q} P_{q}-M_{1}+Q_{1}-Q_{2}+T_{1}^{T} A+A^{T} T_{1}-\rho P_{p}-H_{1}-H_{1}^{T}, \\
& \Theta_{12}^{1}=P_{p}+M_{1}-M_{2}-N_{2}+T_{1}^{T} B \alpha_{p} K C-\tau \rho\left(-M_{1}+M_{2}\right)+H_{6}^{T}-H_{2} \text {, } \\
& \Theta_{12}^{2}=P_{p}+M_{1}-M_{2}-N_{2}+T_{1}^{T} B \alpha_{p} K C+H_{6}^{T}-H_{2} \text {, } \\
& \Theta_{13}=-H_{3}+V \text {, } \\
& \Theta_{14}^{1}=\tau M_{1}-T_{1}^{T}+A^{T} T_{2}-H_{4} \text {, } \\
& \Theta_{14}^{2}=-T_{1}^{T}+A^{T} T_{2}-H_{4} \text {, } \\
& \Theta_{15}=T_{1}^{T} F-H_{5} \text {, } \\
& \Theta_{22}^{1}=-M_{1}+M_{2}+M_{2}^{T}+N_{2}+N_{2}^{T}-\tau \rho\left(M_{1}-M_{2}-M_{2}^{T}\right)+H_{7}+H_{7}^{T} \text {, } \\
& \Theta_{22}^{2}=-M_{1}+M_{2}+M_{2}^{T}+N_{2}+N_{2}^{T}-\tau N_{3}+H_{7}+H_{7}^{T} \text {, } \\
& \Theta_{24}^{1}=-M_{1}^{T}+\tau\left(M_{2}^{T}+N_{2}^{T}\right)+\left(T_{2}^{T} B \alpha K C\right)^{T}+H_{9} \text {, } \\
& \Theta_{24}^{2}=-M_{1}^{T}+\left(T_{2}^{T} B \alpha K C\right)^{T}+H_{9} \text {, } \\
& \Theta_{33}=-Q_{1}-Q_{2} \text {, } \\
& \Theta_{44}^{1}=\tau N_{1}+\tau^{2} Q_{2}-T_{2}^{T}-T_{2} \text {, } \\
& \Theta_{44}^{2}=\tau^{2} Q_{2}-T_{2}^{T}-T_{2} \text {, } \\
& \Theta_{44}^{1}=\tau N_{1}+\tau^{2} Q_{2}-T_{2}^{T}-T_{2} \text {, } \\
& \Theta_{44}^{2}=\tau^{2} Q_{2}-T_{2}^{T}-T_{2} \text {, } \\
& \psi \triangleq \max _{p \in \mathcal{S}}\left\{\lambda_{\max }\left(\widetilde{P}_{p}\right)\right\}+\lambda_{\max }\left(\widetilde{Q}_{1}\right)+\lambda_{\max }\left(\widetilde{Q}_{2}\right) \text {, } \\
& \widetilde{P}_{p}=R^{-(1 / 2)} Q_{p} R^{-(1 / 2)} \text {, } \\
& \widetilde{Q}_{s}=R^{-(1 / 2)} Q_{s} R^{-(1 / 2)}, \quad s=1,2 \text {. }
\end{aligned}
$$


Proof. Establishing a Lyapunov functional as $V\left(t, r_{t}\right)=\sum_{i=1}^{4} V_{i}\left(t, r_{t}\right)$, where

$$
\begin{aligned}
& V_{1}\left(t, r_{t}\right)=\delta^{T}(t) P\left(r_{t}\right) \delta(t) \\
& V_{2}\left(t, r_{t}\right)=\left(\tau-\left(t-t_{k}\right)\right) \vartheta_{1}^{T}(t) \mathscr{M} \vartheta_{1}(t) \\
& V_{3}\left(t, r_{t}\right)=\left(\tau-\left(t-t_{k}\right)\right) \int_{t_{k}}^{t} e^{\rho(t-s)} \vartheta_{2}^{T}\left(s, t_{k}\right) \mathcal{N} \vartheta_{2}\left(s, t_{k}\right) \mathrm{d} s, \\
& V_{4}\left(t, r_{t}\right)=\int_{t-\tau}^{t} e^{\rho(t-s)} \delta^{T}(s) Q_{1} \delta(s) \mathrm{d} s+\tau \int_{-\tau}^{0} \int_{t+\theta}^{t} e^{\rho(t-s)} \dot{\delta}^{T}(s) Q_{2} \dot{\delta}(s) \mathrm{d} s \mathrm{~d} \theta
\end{aligned}
$$

where

$$
\begin{aligned}
\vartheta_{1}(t) & =\left[\begin{array}{ll}
\delta^{T}(t) & \delta^{T}\left(t_{k}\right)
\end{array}\right]^{T}, \\
\vartheta_{2}\left(s, t_{k}\right) & =\left[\begin{array}{ll}
\dot{\delta}^{T}(s) & \delta^{T}\left(t_{k}\right)
\end{array}\right]^{T}, \\
\mathscr{M} & =\left[\begin{array}{cc}
M_{1} & -M_{1}+M_{2} \\
* & M_{1}-M_{2}-M_{2}^{T}
\end{array}\right], \\
\mathcal{N} & =\left[\begin{array}{cc}
N_{1} & N_{2} \\
* & N_{3}
\end{array}\right] .
\end{aligned}
$$

The weak infinitesimal operator $\mathscr{L}\{\cdot\}$ can be inferred as $\mathscr{L} V\left(t, r_{t}\right)=\frac{\partial V\left(t, r_{t}\right)}{\partial t}+\dot{\delta}^{T}(t) \frac{\partial V\left(t, r_{t}\right)}{\partial t}+\sum_{q=\epsilon \mathcal{S}} \pi_{p q} P_{q} V\left(t, r_{t}\right)$

Applying the operator $\mathscr{L}\{\cdot\}$ along the power system (10), which yields

$$
\begin{aligned}
\mathscr{L} V_{1}\left(t, r_{t}\right)= & \delta^{T}(t) P_{p} \dot{\delta}(t)+\dot{\delta}^{T}(t) P \delta(t)+\delta^{T}(t) \sum_{q=1}^{S} \pi_{p q} P_{q} \delta(t) \\
\mathscr{L} V_{2}\left(t, r_{t}\right)= & \left(\tau-\left(t-t_{k}\right)\right) \vartheta_{1}^{T}(t) \mathscr{M}\left[\begin{array}{ll}
\dot{\delta}^{T}(t) & 0
\end{array}\right]^{T}+\left(\tau-\left(t-t_{k}\right)\right)\left[\begin{array}{ll}
\dot{\delta}(t) & 0
\end{array}\right]^{T} \mathscr{M}_{1}(t)-\vartheta_{1}^{T}(t) \mathscr{M}_{1}(t), \\
\mathscr{L} V_{3}\left(t, r_{t}\right) \leq & -\int_{t_{k}}^{t} \dot{\delta}^{T}(s) N_{1} \dot{\delta}(s) \mathrm{d} s-2 \delta^{T}(t) U_{2} \delta\left(t_{k}\right)+\delta^{T}\left(t_{k}\right)\left(N_{2}+N_{2}^{T}-\left(t-t_{k}\right) N_{3}\right) \delta\left(t_{k}\right) \\
& +\left(\tau-\left(t-t_{k}\right)\right) \dot{\delta}^{T}(t) N_{1} \dot{\delta}(t)+2\left(\tau-\left(t-t_{k}\right)\right) \dot{\delta}^{T}(t) N_{2} \delta\left(t_{k}\right)+\rho V_{3}\left(t, r_{t}\right) \\
\mathscr{L} V_{4}\left(t, r_{t}\right) \leq & \delta^{T}(t) Q_{1} \delta(t)-\delta^{T}(t-\tau) Q_{1} \delta(t-\tau)+\tau^{2} \dot{\delta}^{T}(t) Q_{2} \dot{\delta}(t)-\tau \int_{t-\tau}^{t} \dot{\delta}^{T}(s) Q_{2} \dot{\delta}(s) \mathrm{d} s+\rho V_{4}\left(t, r_{t}\right)
\end{aligned}
$$

Based on Lemma 1, the following inequality can be devised:

$$
\begin{aligned}
& -\int_{t_{k}}^{t} \dot{\delta}^{T}(s) N_{1} \dot{\delta}(s) \mathrm{d} s \leq-\frac{1}{t-t_{k}} \xi^{T}(t) \mathscr{W}_{1}^{\mathcal{T}} \mathscr{Q}_{1} \mathscr{W}_{1} \xi(t), \\
& -\tau \int_{t-\tau}^{t} \dot{\delta}^{T}(s) Q_{2} \dot{\delta}(s) \mathrm{d} s \leq-\xi^{T}(t) \mathscr{W}_{2}^{\mathcal{T}} \mathscr{Q}_{2} \mathscr{W}_{2} \xi(t)
\end{aligned}
$$

where

$$
\begin{aligned}
& \xi^{T}(t)=\left[\begin{array}{lllll}
\delta^{T}(t) & \delta^{T}\left(t_{k}\right) & \delta^{T}(t-\tau) & \dot{\delta}(t) & \omega^{T}(t)
\end{array}\right], \\
& \mathscr{W}_{1}=\left[\begin{array}{ccccc}
I & 0 & 0 & 0 & 0 \\
0 & -I & 0 & 0 & 0
\end{array}\right] \text {, } \\
& \mathscr{W}_{2}=\left[\begin{array}{ccccc}
I & 0 & 0 & 0 & 0 \\
0 & 0 & -I & 0 & 0
\end{array}\right] \text {. }
\end{aligned}
$$

It is well known that for any matrices $\mathscr{H}$, one gets

$\frac{1}{t-t_{k}}\left(\mathscr{Q}_{1} \mathscr{W}_{1}-\left(t-t_{k}\right) \mathscr{H}\right)^{T} \mathscr{Q}_{1}^{-1}\left(\mathscr{Q}_{1} \mathscr{W}_{1}-\left(t-t_{k}\right) \mathscr{H}\right) \geq 0$

The aforementioned condition can be rewritten as $-\frac{1}{t-t_{k}} \mathscr{W}_{1}^{T} \mathscr{Q}_{1} \mathscr{W}_{1} \leq-\mathscr{H}^{T} \mathscr{W}_{1}-\mathscr{W}_{1}^{T} \mathscr{H}+\left(t-t_{k}\right) \mathscr{H}^{T} \mathscr{Q}_{1}^{-1} \mathscr{H}$. that

On the other hand, for any matrices $T_{1}$ and $T_{2}$, it is clear

$$
\begin{aligned}
0= & 2\left(\delta^{T}(t) T_{1}^{T}+\dot{\delta}^{T}(t) T_{2}^{T}\right)[-\dot{\delta}(t)+A \delta(t) \\
& \left.+B \alpha_{p} K C \delta\left(t_{k}\right)+F \omega(t)\right] .
\end{aligned}
$$


Substituting (23)-(32) into (20), it can be deduced that

$$
\mathrm{E}\left\{\mathscr{L} V\left(t, r_{t}\right)\right\}-\rho V\left(t, r_{t}\right)-\gamma^{2} \omega^{T}(t) \omega(t) \leq \xi^{T}(t)\left[\Theta(t)+\left(t-t_{k}\right) \mathscr{H}^{T} Q_{1}^{-1} \mathscr{H}\right] \xi(t), \quad t \in\left[t_{k}, t_{k+1}\right)
$$

where

$$
\begin{aligned}
& \Theta(t)=\left[\begin{array}{ccccc}
\Theta_{11} & \Theta_{12} & \Theta_{13} & \Theta_{14} & \Theta_{15} \\
* & \Theta_{22} & H_{8}^{T} & \Theta_{24} & H_{10} \\
* & * & \Theta_{33} & 0 & 0 \\
* & * & * & \Theta_{44} & T_{2}^{T} F \\
* & * & * & * & -\gamma^{2} I
\end{array}\right] \\
& \Theta_{11}=\sum_{q=1}^{S} \pi_{p q} P_{q}-M_{1}+Q_{1}-Q_{2}+T_{1}^{T} A+A^{T} T_{1}-\rho P_{p}-\left(\tau-\left(t-t_{k}\right)\right) \rho M_{1}-H_{1}-H_{1}^{T}, \\
& \Theta_{12}=P_{p}+M_{1}-M_{2}-N_{2}+T_{1}^{T} B \alpha_{p} K C-\left(\tau-\left(t-t_{k}\right)\right) \rho\left(-M_{1}+M_{2}\right)+H_{6}^{T}-H_{2}, \\
& \Theta_{13}=-H_{3}+V, \\
& \Theta_{14}=\left(\tau-\left(t-t_{k}\right)\right) M_{1}-T_{1}^{T}+A^{T} T_{2}-H_{4}, \\
& \Theta_{15}=T_{1}^{T} F-H_{5}, \\
& \Theta_{22}=-M_{1}+M_{2}+M_{2}^{T}+N_{2}+N_{2}^{T}-\left(t-t_{k}\right) N_{3}-\left(\tau-\left(t-t_{k}\right)\right) \rho\left(M_{1}-M_{2}-M_{2}^{T}\right)+H_{7}+H_{7}^{T}, \\
& \Theta_{24}=-M_{1}^{T}+\left(\tau-\left(t-t_{k}\right)\right)\left(M_{2}^{T}+N_{2}^{T}\right)+\left(T_{2}^{T} B \alpha K C\right)^{T}+H_{9}, \\
& \Theta_{33}=-Q_{1}-Q_{1}, \\
& \Theta_{44}=\left(\tau-\left(t-t_{k}\right)\right) N_{1}+\tau^{2} Q_{2}-T_{2}^{T}-T_{2} .
\end{aligned}
$$

Note that (33) is a convex combination of $t-t_{k}$ and $\tau-\left(t-t_{k}\right)$, in accordance with Schur complement; one can deduce that $\Theta(t)+\left(t-t_{k}\right) \mathscr{H}^{T} Q_{1}^{-1} \mathscr{H}<0$ if and only if (15) and (16) hold. Therefore, one can see that

$$
\mathrm{E}\left\{\mathscr{L} V\left(t, r_{t}\right)\right\}<\rho V\left(t, r_{t}\right)+\gamma^{2} \omega^{T}(t) \omega(t), \quad t \in\left[t_{k}, t_{k+1}\right)
$$

By integrating the both sides of (35) from $t_{k}$ to $t$ and simple derivation, it yields

$$
\begin{aligned}
\mathrm{E}\{V(t, p)\}< & e^{\rho\left(t-t_{k}\right)} \mathrm{E}\left\{V\left(t_{k}, p\right)\right\}+\int_{t_{k}}^{t} e^{\rho(t-s)} \gamma^{2} \omega^{T}(s) \omega(s) \mathrm{d} s \\
\leq & e^{\rho\left(t-t_{k-1}\right)} \mathrm{E}\left\{V\left(t_{k-1}, p\right)\right\}+e^{\rho\left(t-t_{k-1}\right)} \int_{t_{k-1}}^{t_{k}} e^{\rho\left(t_{k}-s\right)} \gamma^{2} \omega^{T}(s) \omega(s) \mathrm{d} s \\
& +\int_{t_{k}}^{t} e^{\rho(t-s)} \gamma^{2} \omega^{T}(s) \omega(s) \mathrm{d} s \leq \cdots \leq e^{t-t_{0}} \mathrm{E}\left\{V\left(t_{0}, p\right)\right\}+e^{\rho\left(t-t_{1}\right)} \int_{t_{0}}^{t_{1}} e^{\rho\left(t_{1}-s\right)} \gamma^{2} \omega^{T}(s) \omega(s) \mathrm{d} s \\
& +\cdots+\int_{t_{k}}^{t} e^{\rho(t-s)} \gamma^{2} \omega^{T}(s) \omega(s) \mathrm{d} s \leq e^{\rho\left(t-t_{0}\right)}\left(\mathrm{E}\left\{V\left(t_{0}, p\right)\right\}+\int_{t_{0}}^{t} \gamma^{2} \omega^{T}(s) \omega(s) \mathrm{d} s\right) \\
\leq & e^{\rho T}\left(\mathrm{E}\left\{V\left(t_{0}, p\right)\right\}+\gamma^{2} \bar{\omega}\right) .
\end{aligned}
$$

Recalling the Lyapunov functional (20), we can get 


$$
\begin{aligned}
\mathrm{E}\left\{V\left(t, r_{t}\right)\right\} & \geq \lambda_{\min }\left(\widetilde{P}_{p}\right) \delta^{T}(t) R \delta(t), \\
\mathrm{E}\left\{V\left(t_{0}, r_{t}\right)\right\} & \leq\left(\max _{p \in \mathcal{S}}\left\{\lambda_{\max }\left(\widetilde{P}_{p}\right)\right\}+\lambda_{\text {max }}\left(Q_{1}\right)+\lambda_{\max }\left(Q_{2}\right)\right) \delta^{T}\left(t_{0}\right) R \delta\left(t_{0}\right) .
\end{aligned}
$$

Substituting (37) and (38) into (36), we can obtain

$$
\inf _{p \in \mathcal{S}}\left\{\lambda_{\min }\left(\widetilde{P}_{p}\right)\right\} \mathrm{E}\left\{\delta^{T}(t) R \delta(t)\right\}<e^{\rho T}\left(\psi c_{1}+\gamma^{2} \bar{\omega}\right) .
$$

In light of (17), it can be concluded from (39) that $\mathscr{E}\left\{\delta^{T}(t) R \delta(t)\right\}<c_{2}$. Thus, from Definition 2, we have to derive that power system (10) is SFTB over the time interval $\left[t_{0}, T\right]$.

In the following, the actuator constraints (18) will be discussed. In light of (9), one has

$$
\begin{aligned}
\left|u_{s}^{F}(t)\right| & \leq\left|u_{s}(t)\right|=\left|K_{s} C \delta\left(t_{k}\right)\right|^{2} \\
& \leq\left|K_{s} C R^{-(1 / 2)}\right|^{2} \times\left|R^{1 / 2} \delta\left(t_{k}\right)\right|^{2} \\
& =K_{s} C R^{-1} C^{T} K_{s}^{T} \delta^{T}\left(t_{k}\right) R \delta\left(t_{k}\right) \\
& \leq c_{2} K_{s} C R^{-1} C^{T} K_{s}^{T} .
\end{aligned}
$$

Recalling Assumption 2, it yields

$$
c_{2} K_{s} C R^{-1} C^{T} K_{s}^{T}<u_{s \max }^{2}, \quad s=1,2, \ldots, n_{u} .
$$

According to Schur complement, (18) can be guaranteed by (41), which completes the proof of Theorem 1 .

Theorem 2. For given parameters $\rho>0, c_{1}>0, c_{2}>0, T>0$, $\bar{\omega}>0, u_{\max }$, and matrix $R$, the closed-loop power system (10) is called SFTB with respect to $\left(c_{1}, c_{2}, \bar{\omega}, T, R\right)$ and meet an $H_{\infty}$ performance index $\bar{\gamma}=\gamma \sqrt{e^{\rho T}}$, if there exists matrix $P_{p}>0$, $N_{1}>0, N_{3}>0, Q_{1}>0, Q_{2}>0$, and matrices $X, Y$ with suitable dimensions, such that $\forall p \in \mathcal{S}$

$$
\begin{aligned}
& {\left[\begin{array}{ccc}
\bar{\Theta}^{1} & \mathscr{Z}_{1} & \mathscr{Z}_{2} \\
* & -\varepsilon_{1}\left(X+X^{T}\right) & 0 \\
* & * & -\varepsilon_{2}\left(X+X^{T}\right)
\end{array}\right]<0,} \\
& {\left[\begin{array}{cccc}
\bar{\Theta}^{2} & \tau \mathscr{H}^{T} & \mathscr{Z}_{1} & \mathscr{Z}_{2} \\
* & -\tau \mathscr{Q}_{1}^{-1} & 0 & 0 \\
* & * & -\varepsilon_{1}\left(X+X^{T}\right) & 0 \\
* & * & * & -\varepsilon_{2}\left(X+X^{T}\right)
\end{array}\right]<0,} \\
& e^{\rho T} \bar{\psi} c_{1}+e^{\rho T} \gamma^{2} \bar{\omega}<\lambda_{1} c_{2}, \\
& \lambda_{1} R<P_{p}<\lambda_{2} R, \quad Q_{1}<\lambda_{3} R, \quad Q_{2}<\lambda_{4} R, \\
& {\left[\begin{array}{ccc}
-u_{s \max }^{2} & Y C & I-X \\
* & -\frac{R}{c_{2}} & \varepsilon_{3} C^{T} Y^{T} \\
* & * & -\varepsilon_{3}\left(X+X^{T}\right)
\end{array}\right]<0, \quad s=1,2, \ldots, n_{u},}
\end{aligned}
$$

where 


$$
\begin{aligned}
& \Theta^{i}=\left[\begin{array}{ccccc}
\bar{\Theta}_{11}^{i} & \Theta_{12}^{i} & \Theta_{13} & \Theta_{14}^{i} & \Theta_{15} \\
* & \Theta_{22}^{i} & H_{8}^{T} & \Theta_{24}^{i} & H_{10} \\
* & * & \Theta_{33} & 0 & 0 \\
* & * & * & \Theta_{44}^{i} & T_{2}^{T} F \\
* & * & * & * & -\gamma^{2} I
\end{array}\right], \\
& \bar{\psi} \triangleq \lambda_{2}+\tau \lambda_{3}+\tau^{2} \lambda_{4} \\
& \bar{\Theta}_{11}^{1}=\sum_{q=1}^{S} \pi_{p q} P_{q}-M_{1}+Q_{1}-Q_{2}+T_{1}^{T} A+A^{T} T_{1}-\rho P_{p}-\tau \rho M_{1}-H_{1}-H_{1}^{T}-C^{T} C, \\
& \bar{\Theta}_{11}^{2}=\sum_{q=1}^{S} \pi_{p q} P_{q}-M_{1}+Q_{1}-Q_{2}+T_{1}^{T} A+A^{T} T_{1}-\rho P_{p}-H_{1}-H_{1}^{T}-C^{T} C, \\
& \mathscr{Z}_{1}=\left[\alpha\left(T_{1}^{T} B-B X\right)^{T} \quad \varepsilon Y C \quad 0000\right]^{T}, \\
& \mathscr{Z}_{2}=\left[\begin{array}{lllll}
Y C & 0 & 0 & \alpha\left(T_{2}^{T} B-B X\right)^{T} & 0
\end{array}\right]^{T} \text {. }
\end{aligned}
$$

The controller gain is deduced as

$$
K=X^{-1} Y \text {. }
$$

Proof. By resorting to the same Lyapunov functional as displayed in (20), and adopting the similar derivations in the proof of Theorem 1 , for $t \in\left[t_{k}, t_{k+1}\right)$, the following inequality can be realized:

$$
\mathrm{E}\left\{\mathscr{L} V\left(t, r_{t}\right)\right\}<\rho V\left(t, r_{t}\right)-y^{T}(t) y(t)+\gamma^{2} \omega^{T}(t) \omega(t), \quad t \in\left[t_{k}, t_{k+1}\right) .
$$

Integrating the both sides of (49) from $t_{k}$ to $t$, it yields

$$
\mathrm{E}\{V(t, p)\}<e^{\rho\left(t-t_{k}\right)} V\left(t_{k}, p\right)+\int_{t_{k}}^{t} e^{\rho(t-s)}\left(-y^{T}(s) y(s)+\gamma^{2} \omega^{T}(s) \omega(s)\right) \mathrm{d} s, \quad t \in\left[t_{k}, t_{k+1}\right)
$$

Clearly, (50) can be rewritten as

$$
\mathrm{E}\{V(t, p)\}<e^{\rho\left(t-t_{0}\right)} V\left(t_{0}, p\right)+\int_{t_{0}}^{t} e^{\rho(t-s)}\left(-y^{T}(s) y(s)+\gamma^{2} \omega^{T}(s) \omega(s)\right) \mathrm{d} s .
$$

Under the zero-initial conditions, one gets $V\left(t_{0}, p\right)=0$. It follows from (51) that

$$
\int_{t_{0}}^{t} e^{\rho(t-s)} y^{T}(s) y(s) \mathrm{d} s<\int_{t_{0}}^{t} e^{\rho(t-s)} \gamma^{2} \omega^{T}(s) \omega(s) \mathrm{d} s .
$$

Since $1 \leq e^{\rho(t-s)} \leq e^{\rho t}$ and $t \in\left[t_{0}, T\right]$, (52) signifies

$$
\mathrm{E}\left\{\int_{t_{0}}^{t} y^{T}(s) y(s) \mathrm{d} s\right\}<\bar{\gamma}^{2} \int_{t_{0}}^{t} \omega^{T}(s) \omega(s) \mathrm{d} s
$$

with $\bar{\gamma}=\sqrt{e^{\rho T}} \gamma$. Therefore, the $H_{\infty}$ performance index $\bar{\gamma}$ is ensured.
Meanwhile, in light of Lemma 1 of [28], conditions (42) and (43) can be easily attained. Similarly, (46) can be ensured. The proof is completed.

\section{Computational Experiments}

In this example, a single-area power system is studied for simulating the derived results. Similar to [6], the parameters of system (1) are expressed in Table 2.

Taking the actuator faults into consideration, which is characterized by a Markov process, the possible fault coefficients are $\alpha_{1}=1, \alpha_{2}=0.8$, and $\alpha_{3}=0.5$. Furthermore, the transition probability matrix among faults is given by 
TABLe 2: Physical meaning.

\begin{tabular}{ccccccc}
\hline Parameters & $R$ & $D$ & $M(s)$ & $\beta$ & $T_{g}(s)$ & $T_{c h}(s)$ \\
\hline & 0.05 & 1.0 & 10 & 21.0 & 0.1 & 0.3 \\
\hline
\end{tabular}

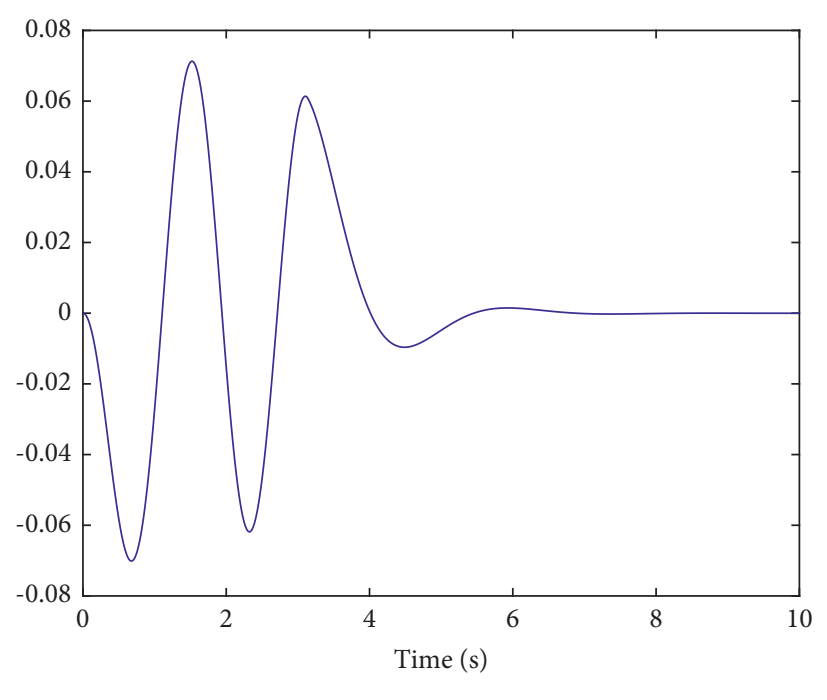

_ Frequency deriation

Figure 2: Evolution of frequency derivation.

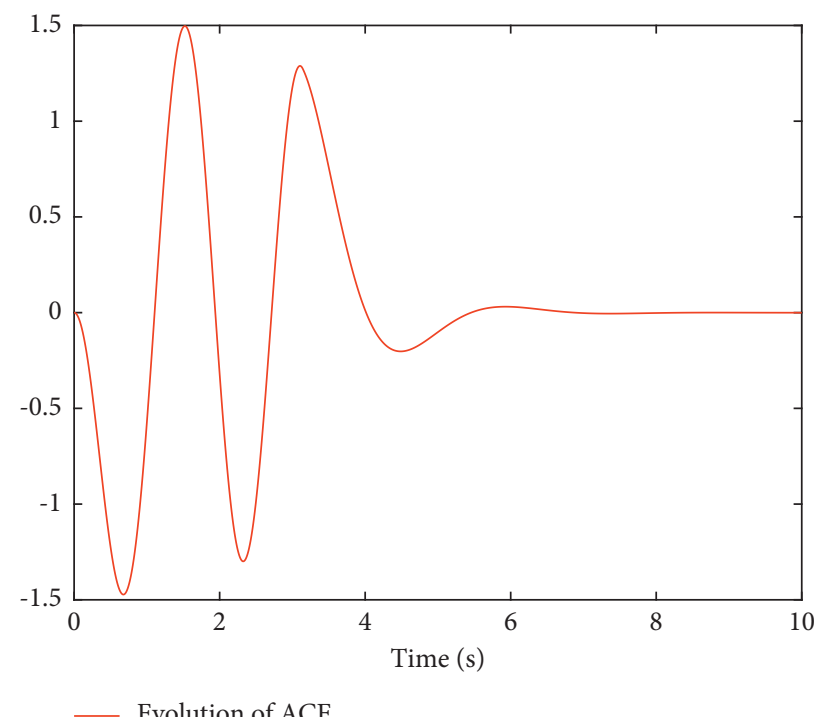

Figure 3: Evolution of ACE.

$$
\Pi=\left[\begin{array}{lll}
0.2 & 0.5 & 0.3 \\
0.8 & 0.1 & 0.1 \\
0.3 & 0.3 & 0.4
\end{array}\right] .
$$

On the other hand, other parameters are selected as $\tau=0.2, \gamma=0.6, \rho=0.2, c_{1}=0.1, c_{2}=1, \bar{\omega}=0.8, R=I_{4 \times 4}$, and $T=8$. The control input $u(t)$ is supposed to be constrained by $|u(t)| \leq u_{\max }=2$. By solving the linear matrix inequalities of Theorem 2, the desired PI-type controller is derived as

$$
K=\left[\begin{array}{ll}
-0.0002 & -0.0183
\end{array}\right]
$$

For graphically verifying the achieved results, we select the initial state disturbance $\omega(t)$ as

$$
\omega(t)= \begin{cases}1.8 \sin (4 t), & \text { if } t \leq \pi \\ 0, & \text { otherwise. }\end{cases}
$$




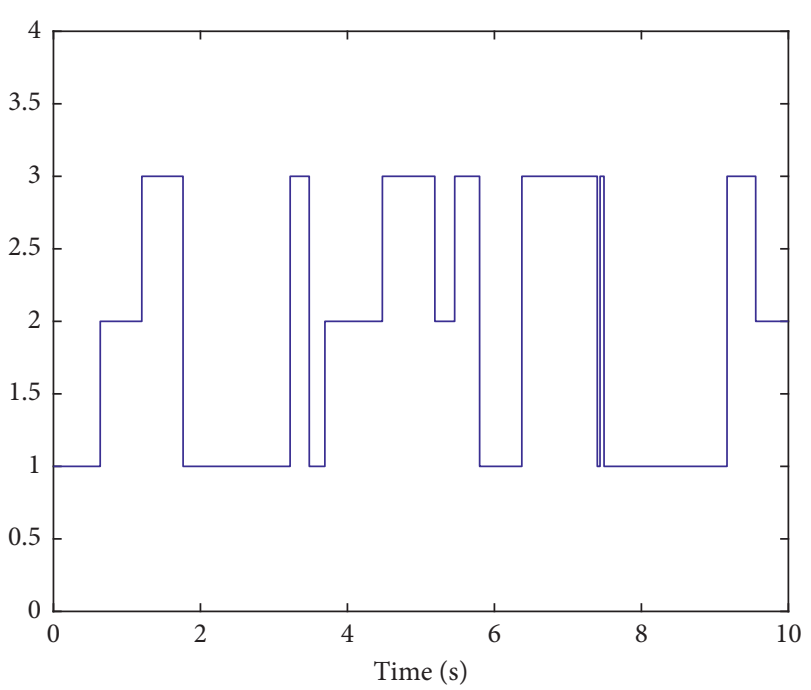

System Modes

FIgURE 4: The mode switching of actuator faults.

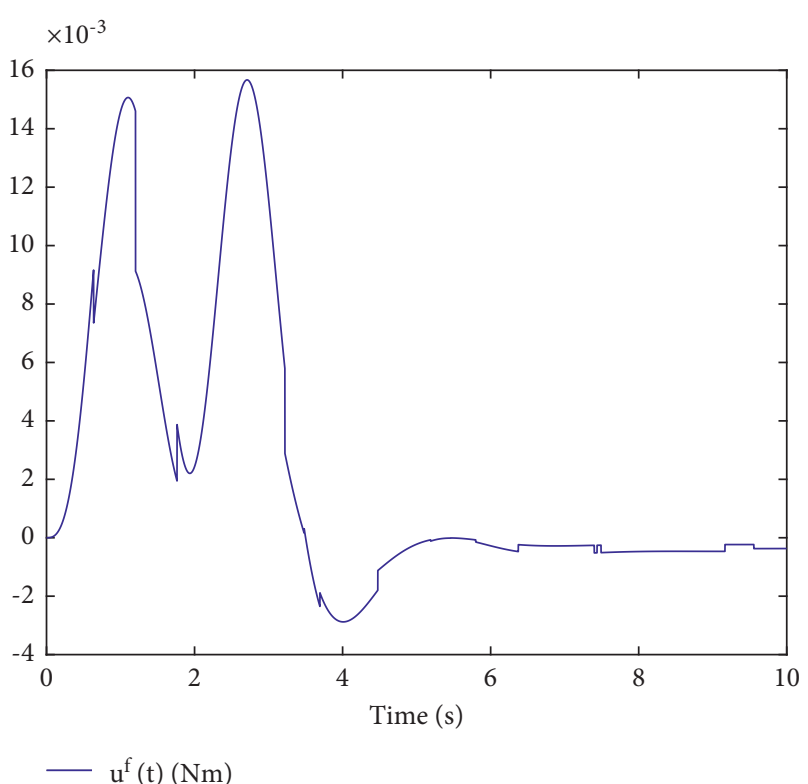

FIGURE 5: Control output.

Added by the aforementioned controller, the simulation results are plotted in Figures 2-7. Figure 2 plots the simulated frequency, and Figure 3 displays the evolution of ACE. Meanwhile, the mode switching of actuator faults is shown in Figure 4, and control output is presented in Figure 5. Furthermore, with the disturbance given in Figure 6, the evolution of $\delta^{T}(t) R \delta(t)$ is expressed in Figure 7. One can be observed from Figure 7 that the state of closedloop system stays in the prefixed region, which implies the resulting system is SFTB. Meanwhile, the input constraint is also satisfied.

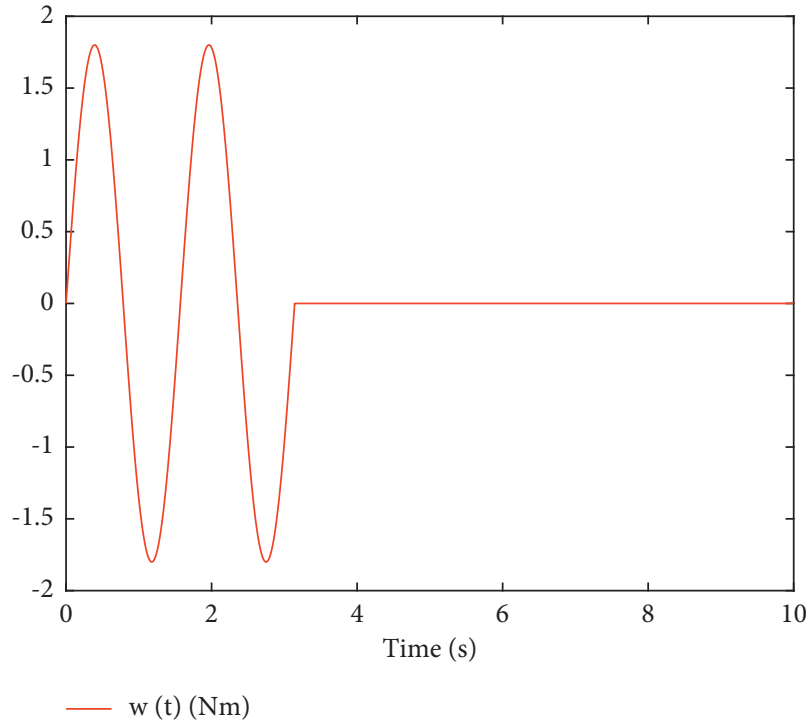

Figure 6: The disturbance $\omega(t)$.

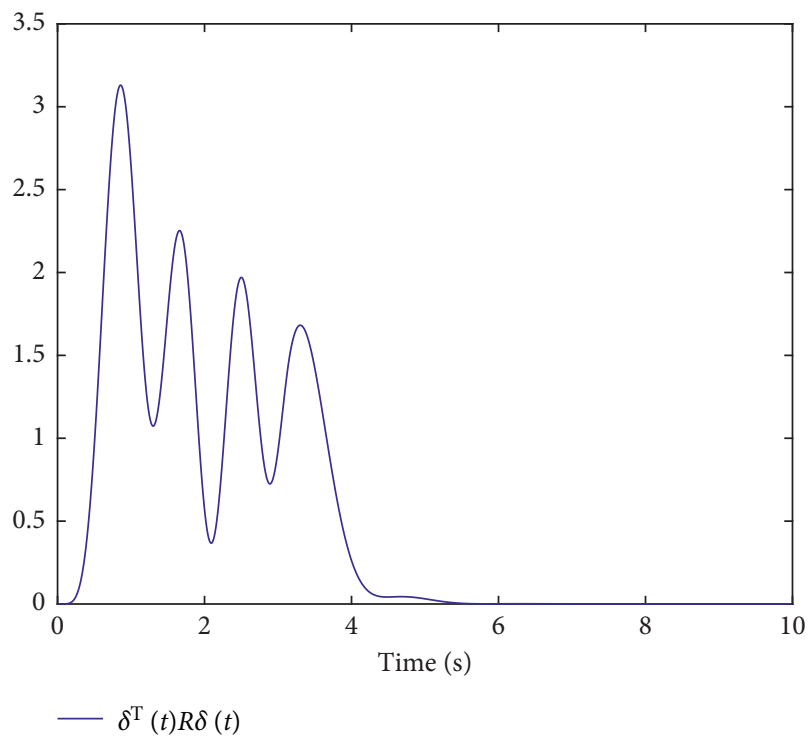

FIgURE 7: The evolution of $\delta^{T}(t) R \delta(t)$.

\section{Conclusions}

In this study, the finite-time LFC problem for power systems with actuator fault has been considered. To better reflect the actual demands of practical dynamics, a generalized framework of the actuator constraint has been studied. Given the randomly occurring actuator fault, a homogeneous Markov chain-based actuator fault has been studied. Together with the piecewise Lyapunov theory, sufficient conditions have been attained. In the end, a numerical example has been applied to verify the effectiveness of the developed results. 


\section{Data Availability}

No data were used to support the current work.

\section{Conflicts of Interest}

The authors declare that they have no conflicts of interest.

\section{References}

[1] J. J. Grainger, W. D. Stevenson, and G. W. Chang, Power System Analysis, McGraw-Hill, New York, NY, USA, 1994.

[2] Y. Wang, R. Zhou, and C. Wen, "Robust load-frequency controller design for power systems," IEEE Proceedings C Generation, Transmission and Distribution, vol. 140, no. 1, pp. 11-16, 1993.

[3] L. Jiang, W. Yao, Q. H. Wu, J. Y. Wen, and S. J. Cheng, "Delaydependent stability for load frequency control with constant and time-varying delays," IEEE Transactions on Power Systems, vol. 27, no. 2, pp. 932-941, 2012.

[4] M. Li and Y. Chen, "A wide-area dynamic damping controller based on robust $H_{\infty}$ control for wide-area power systems with random delay and packet dropout," IEEE Transactions on Power Systems, vol. 33, no. 4, pp. 4026-4037, 2018.

[5] C. Peng and J. Zhang, "Delay-distribution-dependent load frequency control of power systems with probabilistic interval delays," IEEE Transactions on Power Systems, vol. 31, no. 4, pp. 3309-3317, 2016.

[6] S. Kuppusamy and Y. H. Joo, "Resilient reliable $H_{\infty}$ load frequency control of power system with random gain fluctuations," IEEE Transactions on Systems, Man, and Cybernetics: Systems, pp. 1-9, 2021.

[7] H. Zhang, J. Liu, and S. Xu, " $H_{\infty}$ load frequency control of networked power systems via an event-triggered scheme," IEEE Transactions on Industrial Electronics, vol. 67, no. 8, pp. 7104-7113, 2020.

[8] Q. Zhao and J. Jiang, "Reliable state feedback control system design against actuator failures," Automatica, vol. 34, no. 10, pp. 1267-1272, 1998.

[9] K. Zhang, B. Jiang, and M. Staroswiecki, "Dynamic output feedback-fault tolerant controller design for Takagi-Sugeno fuzzy systems with actuator faults," IEEE Transactions on Fuzzy Systems, vol. 18, no. 1, pp. 194-201, 2010.

[10] W. Zhou, J. Fu, H. Yan, X. Du, Y. Wang, and H. Zhou, "Eventtriggered approximate optimal path-following control for unmanned surface vehicles with state constraints," IEEE Transactions on Neural Networks and Learning Systems, pp. 1-15, 2021.

[11] S. Yin, H. Yang, and O. Kaynak, "Sliding mode observer-based FTC for Markovian jump systems with actuator and sensor faults," IEEE Transactions on Automatic Control, vol. 62, no. 7, pp. 3551-3558, 2017.

[12] J. Tao, R. Lu, P. Shi, H. Su, and Z.-G. Wu, "Dissipativity-based reliable control for fuzzy Markov jump systems with actuator faults," IEEE Transactions on Cybernetics, vol. 47, no. 9, pp. 2377-2388, 2017.

[13] Y. Wei, J. Qiu, P. Shi, and L. Wu, "A piecewise-Markovian Lyapunov approach to reliable output feedback control for fuzzy-affine systems with time-delays and actuator faults," IEEE Transactions on Cybernetics, vol. 48, no. 9, pp. 27232735, 2018.

[14] J. Cheng, J. H. Park, X. Zhao, H. R. Karimi, and J. Cao, "Quantized nonstationary filtering of networked Markov switching RSNSs: a multiple hierarchical structure strategy,"
IEEE Transactions on Automatic Control, vol. 65, no. 11, pp. 4816-4823, 2020.

[15] Y. Wang, H. Pu, P. Shi, C. K. Ahn, and J. Luo, "Sliding mode control for singularly perturbed Markov jump descriptor systems with nonlinear perturbation," Automatica, vol. 127, Article ID 109515, 2021.

[16] J. Cheng, J. H. Park, and Z.-G. Wu, "Observer-based asynchronous control of nonlinear systems with dynamic eventbased try-once-discard protocol," IEEE Transactions on Cybernetics, pp. 1-11, 2021.

[17] F. Li, W. X. Zheng, and S. Xu, "Finite-time fuzzy control for nonlinear singularly perturbed systems with input constraints," IEEE Transactions on Fuzzy Systems, p. 1, 2021.

[18] J. Cheng, D. Zhang, W. Qi, J. Cao, and K. Shi, "Finite-time stabilization of T-S fuzzy semi-Markov switching systems: a coupling memory sampled-data control approach," Journal of the Franklin Institute, vol. 357, no. 16, pp. 11256-11280, 2020.

[19] J. Cheng, Y. Wang, J. H. Park, J. Cao, and K. Shi, "Static output feedback quantized control for fuzzy Markovian switching singularly perturbed systems with deception attacks," IEEE Transactions on Fuzzy Systems, p. 1, 2021.

[20] R. Vadivel and Y. H. Joo, "Finite-time sampled-data fuzzy control for a non-linear system using passivity and passification approaches and its application," IET Control Theory \& Applications, vol. 14, no. 8, pp. 1033-1045, 2020.

[21] M. Li and Y. Chen, "Robust tracking control of networked control systems with communication constraints and external disturbance," IEEE Transactions on Industrial Electronics, vol. 64, no. 5, pp. 4037-4047, 2017.

[22] J. Cheng, J. H. Park, J. Cao, and W. Qi, “Asynchronous partially mode-dependent filtering of network-based MSRSNSs with quantized measurement," IEEE Transactions on Cybernetics, vol. 50, no. 8, pp. 3731-3739, 2020.

[23] G. Liu, C. Hua, P. X. Liu, and J. H. Park, "Input-to-state stability for time-delay systems with large delays," IEEE Transactions on Cybernetics, pp. 1-9, 2021.

[24] L. Hetel, C. Fiter, H. Omran et al., "Recent developments on the stability of systems with aperiodic sampling: an overview," Automatica, vol. 76, pp. 309-335, 2017.

[25] D. Zhang, Z. Xu, H. R. Karimi, Q.-G. Wang, and L. Yu, "Distributed $H_{\infty}$ output-feedback control for consensus of heterogeneous linear multiagent systems with aperiodic sampled-data communications," IEEE Transactions on Industrial Electronics, vol. 65, no. 5, pp. 4145-4155, 2018.

[26] S. Xu, G. Sun, Z. Li, and H. Zheng, "Finite-time robust fuzzy control for non-linear Markov jump systems under aperiodic sampling and actuator constraints," IET Control Theory \& Applications, vol. 11, no. 15, pp. 2419-2431, 2017.

[27] P. Park, J. W. Ko, and C. Jeong, "Reciprocally convex approach to stability of systems with time-varying delays," Automatica, vol. 47, no. 1, pp. 235-238, 2011.

[28] S. Dong, M. Fang, and S. Chen, "Extended dissipativity asynchronous static output feedback control of Markov jump systems," Information Sciences, vol. 514, pp. 375-387, 2020. 